International Journal of Applied Mathematics

Volume 29 No. $4 \quad 2016,425-438$

ISSN: 1311-1728 (printed version); ISSN: 1314-8060 (on-line version)

doi: http://dx.doi.org/10.12732/ijam.v29i4.2

\title{
CHARACTERIZATION OF DELTA OPERATOR FOR EULER, BERNOULLI OF SECOND KIND AND MOTT POLYNOMIALS
}

\author{
A. Maheswaran ${ }^{1 \S}$, C. Elango ${ }^{2}$ \\ Department of Mathematical Sciences \\ Cardamom Planters' Association College \\ Bodinayakanur, 625513, Tamilnadu, INDIA
}

\begin{abstract}
The aim of this paper is to study the characterization of delta operator for some Sheffer polynomials. We investigate, within such context, the characterization of the delta operator for the Euler, the Bernoulli of second kind and the Mott polynomials. We can derive many interesting properties of the above polynomials.
\end{abstract}

AMS Subject Classification: 05A10, 05A16, 41A58

Key Words: delta operator, basic polynomial sequences, Euler polynomials, Bernoulli polynomials of second kind, Mott polynomials, Sheffer polynomials

\section{Introduction}

Rota's operator approach to the finite operator calculus is a systematic study of delta operators on the algebra of polynomials. Many interesting identities for Sheffer polynomials are derived in [8] and [2]. Rota [11] serves as an introduction and a guide to the combinatorics theory. It contains a detailed study of delta

Received: May 13, 2016

(C) 2016 Academic Publications

${ }^{\S}$ Correspondence author 
operator, basic polynomial sequence and Sheffer polynomials. Some properties of Bernoulli and Euler are discussed and some interesting identities are derived in a number of papers [3], [14], [12] and [16]. Several identities related to Bernoulli polynomials of second kind are obtained in [13], [5] and [9]. Rainville [10] and Boas and Buck [1] are fully utilized to discuss the classical polynomials set. The definition and the properties of Mott polynomials are discussed in [15].

The aim of the present paper is to propose some results tied to the Sheffer polynomials corresponding to the delta operator. The rest of the paper is organized in three sections. In the second section, we give some known definitions and theorems from G.C. Rota [11]. The third section deals with discussion of sequential representation of the delta operator [6]. This sequence is unique for any delta operator and this representation plays a vital role in deriving many propositions for the Sheffer polynomials. In the fourth section, we consider the Euler, the Bernoulli of second kind and the Mott polynomials and obtain some interesting propositions for these polynomials. Finally a table which include the characterization of delta operator for these Sheffer polynomials is incorporated.

\section{Preliminaries}

The Euler and the Bernoulli polynomials possess many interesting results and arising in many areas of Mathematics and Physics. The objective of Rota [11] was a unified theory of special polynomials. In this section, we recall terminology, notation, some basic definitions and results of the finite operator calculus, as it has been introduced by Rota [11].

Let $p(x)$ be a polynomial in one variable defined over a real number field $F$ of characteristic zero. The set of such polynomials is denoted by $P$. A sequence of polynomials is $\left\{p_{n}(x) / n \in \mathbb{Z}^{+} \cup\{0\}\right\}$, where $p_{n}(x)$ is exactly of degree $n$.

Definition 1. i. An operator $E^{a}$ is said to be a shift operator if $E^{a} p(x)=$ $p(x+a)$, for all polynomials $p(x)$ in one variable defined over the field $F$ and $a \in F$.

ii. A linear operator $T$ which commutes with all shift operators is called a shift invariant.

In symbols, $T E^{a}=E^{a} T, \quad \forall a \in F$.

iii. A delta operator usually denoted by the letter $Q$, is a shift-invariant operator for which $Q x$ is a non zero constant.

Thus every delta operator $Q$ is a shift invariant. But a shift invariant operator need not be a delta operator. 
The forward difference operator

$$
(\Delta f)(x)=f(x+1)-f(x)
$$

is a delta operator.

Definition 2. Let $Q$ be a delta operator, A polynomial sequence $p_{n}(x)$ is called the sequence of basic polynomials for $Q$ if:

i) $p_{0}(x)=1$;

ii) $p_{n}(0)=0$, whenever $n>0$;

iii) $Q p_{n}(x)=n p_{n-1}(x)$.

The basic polynomials are a large class of polynomial sequences that include the monomials $\left\{x^{n} ; n=0,1,2, \cdots\right\}$, the sequences of Lower factorials $[x]_{n}$, Upper factorials $[x]^{n}$, the Abel polynomials and many others.

The proofs of the following results are skipped. But they are easily read from the reference G.C. Rota [11].

The delta operators possess many of the properties of the usual derivative $D$. The following theorems are good examples.

Theorem 1. (a) Every delta operator has a unique sequence of basic polynomials.

(b) If $Q$ is a delta operator, then $Q a=0$ for every constant $a$.

(c) If $p(x)$ is a polynomial of degree $n$, then $Q p(x)$ is a polynomial of degree $n-1$.

Definition 3. A polynomial sequence $s_{n}(x)$ is called a Sheffer set or a set of Sheffer polynomials for the delta operator $Q$, if:

1. $s_{0}(x)=c \neq 0$,

2. $Q s_{n}(x)=n s_{n-1}(x)$.

Thus from Definitions 2 and 3, every sequence of basic polynomials is a Sheffer polynomial. But the Sheffer polynomials need not be a sequence of basic polynomials.

Using the above definition, Rota [11] gives a theorem which gives a necessary and sufficient condition for Sheffer polynomials relative to $Q$.

Theorem 2. Let $Q$ be a delta operator with basic polynomial set $q_{n}(x)$. Then $s_{n}(x)$ is a Sheffer set relative to $Q$ if and only if there exists an invertible shift invariant operator $S$ such that $s_{n}(x)=S^{-1} q_{n}(x)$. 


\section{Sequential Representation of Delta Operator $Q$}

Using the expressions for $Q\left(x^{2}\right), Q\left(x^{3}\right), Q\left(x^{4}\right), \cdots$, we attempt to formulate the delta operator in terms of a sequence of real numbers. By Theorem 1 and the definition of the basic polynomials, we obtain the following theorem.

Theorem 3. For the monomials $\left\{x^{n}: n \in \mathbb{Z}^{+} \cup\{0\}\right\}$, and for each $\alpha_{r}$ an arbitrary real number,

$$
Q\left(x^{n}\right)=\sum_{r=1}^{n}\left(\begin{array}{l}
n \\
r
\end{array}\right) \alpha_{r} x^{n-r}
$$

Proof. Take $Q(x)=\alpha_{1} \neq 0$ and construct $Q\left(x^{2}\right)=c_{0} x+c_{1}$. Since $Q$ is shift invariant, we have $E^{a} Q\left(x^{2}\right)=Q E^{a}\left(x^{2}\right)$. Solving, we get $c_{0}=2 \alpha_{1}$ and $c_{1}$ is a new independent constant which may be taken as $\alpha_{2}$. Hence $Q\left(x^{2}\right)=2 \alpha_{1} x+\alpha_{2}$. Thus the theorem is true for $n=1$ and 2 .

Let us assume that the result is true for all $n=k$.

Therefore,

$$
\begin{aligned}
& Q\left(x^{k}\right)=\sum_{r=1}^{k}\left(\begin{array}{l}
k \\
r
\end{array}\right) \alpha_{r} x^{k-r} \\
= & \left(\begin{array}{l}
k \\
1
\end{array}\right) \alpha_{1} x^{k-1}+\left(\begin{array}{l}
k \\
2
\end{array}\right) \alpha_{2} x^{k-2}+\cdots+\left(\begin{array}{l}
k \\
r
\end{array}\right) \alpha_{r} x^{k-r}+\cdots+\alpha_{k} .
\end{aligned}
$$

Since $\left\{x^{n}\right\}$ is a basic polynomial sequence, it satisfies $Q p_{n}(x)=n p_{n-1}(x)$ and hence, we have

$$
Q\left(x^{k}\right)=k x^{k-1}
$$

From (3), we see that the delta operator $Q$ is a usual derivative $D$.

From (2) and (3), we get

$$
\left(\begin{array}{l}
k \\
1
\end{array}\right) \alpha_{1} x^{k-1}+\left(\begin{array}{l}
k \\
2
\end{array}\right) \alpha_{2} x^{k-2}+\cdots+\left(\begin{array}{l}
k \\
r
\end{array}\right) \alpha_{r} x^{k-r}+\cdots+\alpha_{k}=k x^{k-1}
$$

By comparing the corresponding terms, we have $\alpha_{1}=1$ and $\alpha_{j}=0, j=$ $2,3, \cdots, k$.

Therefore, the result is true for $n=k$, and this means that

$$
\alpha_{1}=1 \text { and } \alpha_{j}=0 \quad(j=2,3, \cdots k) \text {. }
$$

Now we have to show that this result is true for $n=k+1$ :

$$
Q\left(x^{k+1}\right)=Q\left(x^{k} x\right)=Q\left(x^{k}\right) x+Q(x) x^{k}=(k+1) x^{k} .
$$


Thus we have

$$
Q\left(x^{k+1}\right)=(k+1) x^{k} .
$$

On other hand, using the property that $Q p_{n}(x)=n p_{n-1}(x)$, we have

$$
Q\left(x^{k+1}\right)=(k+1) p_{k}(x)=(k+1) x^{k} .
$$

From equations (6) and (7), we conclude that the result is true for all $n=k+1$. Thus we proved Theorem 3 .

Here, $Q\left(x^{n}\right)$ has $n$ independent parameters, $\alpha_{i}(i=1,2,3 \ldots n)$. These parameters are unique. We conclude that any delta operator may be fixed uniquely by equation (1).

To study the delta operator, we need analyze only this sequential representation in equation (1).

\begin{tabular}{|l|l|}
\hline$n$ & $Q\left(x^{n}\right)$ \\
\hline 1 & $1 \alpha_{1}$ \\
\hline 2 & $2 \alpha_{1} x+1 \alpha_{2}$ \\
\hline 3 & $3 \alpha_{1} x^{2}+3 \alpha_{2} x+1 \alpha_{3}$ \\
\hline 4 & $4 \alpha_{1} x^{3}+6 \alpha_{2} x^{2}+4 \alpha_{3} x+1 \alpha_{4}$ \\
\hline 5 & $5 \alpha_{1} x^{4}+10 \alpha_{2} x^{3}+10 \alpha_{3} x^{2}+5 \alpha_{4} x+1 \alpha_{5}$ \\
\hline 6 & $6 \alpha_{1} x^{5}+15 \alpha_{2} x^{4}+20 \alpha_{3} x^{3}+15 \alpha_{4} x^{2}+6 \alpha_{5} x+1 \alpha_{6}$ \\
\hline 7 & $7 \alpha_{1} x^{6}+21 \alpha_{2} x^{5}+35 \alpha_{3} x^{4}+35 \alpha_{4} x^{3}+21 \alpha_{5} x^{2}+7 \alpha_{6} x^{5}+1 \alpha_{7}$ \\
\hline
\end{tabular}

Table 1. First few polynomials $Q\left(x^{n}\right)$, for each degree $n$.

The coefficients of $Q\left(x^{n}\right)$ are arranged by a triangular array, say delta triangle is given below

$\begin{array}{cccccccccccc} & & & & 1 & & & & & \\ & & & 2 & & 1 & & & & \\ & & & 3 & & 3 & & 1 & & & \\ & & 4 & & 6 & & 4 & & 1 & & \\ & 5 & & 10 & & 10 & & 5 & & 1 & \\ 6 & & 15 & & 20 & & 15 & & 6 & & 1 \\ \ldots & & & & & & & & & & \end{array}$

Similar to the Pascal triangle, it is also a triangular arrangements of rows. The tip of the triangle is number 1 which makes up the first row. In Pascal triangle, each row, except first, begins and ends with a "1". But in delta triangle, the consecutive rows begins with numbers $1,2,3, \ldots$ respectively but 
ending with 1s. From the second row, the "Pascal Triangle sum" result holds good.

Equation (1) in Theorem 3 is important in deriving many results for Sheffer polynomials. The characterization of the delta operator is determined by the values of $\alpha_{i}^{\prime} s(i=1,2,3 \cdots n)$. In the next section, we study more about the delta operator in particular, the characterization of a delta operator which corresponds to a given sequence of Sheffer polynomials.

Remark 1. A new form of Newton binomial is discussed in [4]. By this method, equation (1) can be written as:

$$
Q\left(x^{n}\right)=\sum_{r=1}^{n} \frac{1}{r !} \alpha_{r} D^{r} x^{n} .
$$

Therefore,

$$
Q \equiv \sum_{r=1}^{n} \frac{1}{r !} \alpha_{r} D^{r}
$$

Putting $p_{n}(x)=p_{n}$ and $D^{r} p_{n}(x)=p_{n}^{(r)}$, we have

$$
Q\left(p_{n}\right)=\sum_{r=1}^{n} \frac{1}{r !} \alpha_{r} p_{n}^{(r)} .
$$

By (iii) in Definition 2, the above equation (10) becomes

$$
Q\left(p_{n}\right)=\sum_{r=1}^{n} \frac{1}{r !} \alpha_{r} p_{n}^{(r)}=n p_{n-1} .
$$

\section{Characterization of Delta Operator for Some Sheffer Polynomials}

(i) The Euler Polynomials. The Euler polynomials can be defined by means of the generating function such that

$$
\frac{2 e^{x t}}{e^{t}+1}=\sum_{n=0}^{\infty} E_{n}(x) \frac{t^{n}}{n !} .
$$

The $n$th Euler polynomial $E_{n}(x)$ is defined as follows:

$$
E_{n}(x)=\sum_{k=0}^{n}\left(\begin{array}{l}
n \\
k
\end{array}\right) \frac{E_{k}}{2^{k}}\left(x-\frac{1}{2}\right)^{n-k} .
$$




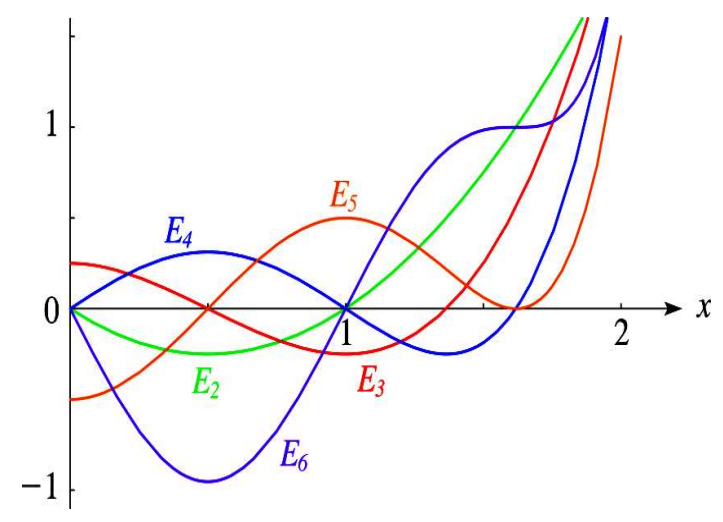

Here, $E_{k}, k=0,1,2 \cdots$ are Euler numbers.

It satisfies $E_{0}(x)=1 \neq 0$ and hence it is a Sheffer set.

The first few Euler polynomials are:

$$
\begin{aligned}
& E_{1}(x)=x-\frac{1}{2} \\
& E_{2}(x)=x^{2}-x \\
& E_{3}(x)=x^{3}-\frac{3}{2} x^{2}+\frac{1}{4} \\
& E_{4}(x)=x^{4}-2 x^{3}+x \\
& E_{5}(x)=x^{5}-\frac{5}{2} x^{4}+\frac{5}{3} x^{2}-\frac{1}{2}
\end{aligned}
$$

For $n=1$,

$$
Q E_{n}=n E_{n-1} \text { becomes } Q E_{1}=1 E_{0} \text {. }
$$

From Table 1,

$$
Q E_{1}=\alpha_{1} \text { and } 1 E_{0}=1 \Rightarrow \alpha_{1}=1
$$

For $n=2$,

$$
Q E_{n}=n E_{n-1} \text { becomes } Q E_{2}=2 E_{1} \text {. }
$$

By Table 1,

$$
Q E_{2}=2 \alpha_{1} x+\alpha_{2}-\alpha_{1} \text { and } 2 E_{1}=2 x-1 \Rightarrow \alpha_{1}=1 \& \alpha_{2}=0 .
$$

For $n=3$,

$$
Q E_{n}=n E_{n-1} \text { becomes } Q E_{3}=3 E_{2} .
$$

From (1) in Theorem 3,

$$
Q E_{3}=\left(3 \alpha_{1}\right) x^{2}+\left(3 \alpha_{2}-3 \alpha_{1}\right) x+\alpha_{3}-\frac{3}{2} \alpha_{2} \text { and } 3 E_{2}=3 x^{2}-3 x \text {. }
$$

Equating the corresponding terms, we get

$$
\alpha_{1}=1, \alpha_{2}=0 \& \alpha_{3}=0 .
$$


By a similar procedure, we get

$$
\alpha_{1}=1 \text { and } \alpha_{r}=0 \text { for all } r \geq 2 .
$$

Hence the characterization of the delta operator for Euler polynomials being $\alpha_{1}=1$, and $\alpha_{r}=0$ for all $r \geq 2$.

Thus we obtain the following proposition.

Proposition 1. For the Euler polynomial

$$
E_{n}(x)=\sum_{k=0}^{n}\left(\begin{array}{l}
n \\
k
\end{array}\right) \frac{E_{k}}{2^{k}}\left(x-\frac{1}{2}\right)^{n-k},
$$

the characterization of delta operator being $\alpha_{1}=1$, and $\alpha_{r}=0$ for all $r \geq 2$.

Remark 2. Here, $Q\left(x^{n}\right)=n x^{n-1}$.

(ii) Bernoulli Polynomials. The Bernoulli polynomials of second kind are defined by the following generating function

$$
\sum_{n=0}^{\infty} b_{n}(x) \frac{t^{n}}{n !}=\frac{t}{\log (1+t)}(1+t)^{x} .
$$

Roman (1984) defined the Bernoulli numbers of the second kind as $b_{n}=$ $b_{n}(0)$. The first few Bernoulli numbers $b_{n}$ of the second kinds are:

$$
b_{0}=1, b_{1}=\frac{1}{2}, b_{2}=\frac{-1}{12}, b_{3}=\frac{1}{24}, b_{4}=\frac{-19}{720}, b_{5}=\frac{3}{160}, \cdots .
$$

By (12), we easily get

$$
b_{n}(x)=\sum_{k=0}^{n}\left(\begin{array}{l}
n \\
k
\end{array}\right) b_{k}[x]_{n-k}
$$

where $[x]_{n}=x(x-1)(x-2) \cdots(x-n+1), n \geq 0$.

The first few Bernoulli polynomials of the second kind are:

$b_{0}(x)=1$

$b_{1}(x)=\frac{1}{2}(2 x+1)$

$b_{2}(x)=\frac{1}{6}\left(6 x^{2}-1\right)$

$b_{3}(x)=\frac{1}{4}\left(4 x^{3}-6 x^{2}+1\right)$

$b_{4}(x)=\frac{1}{30}\left(30 x^{4}-120 x^{3}+120 x^{2}-19\right)$ 


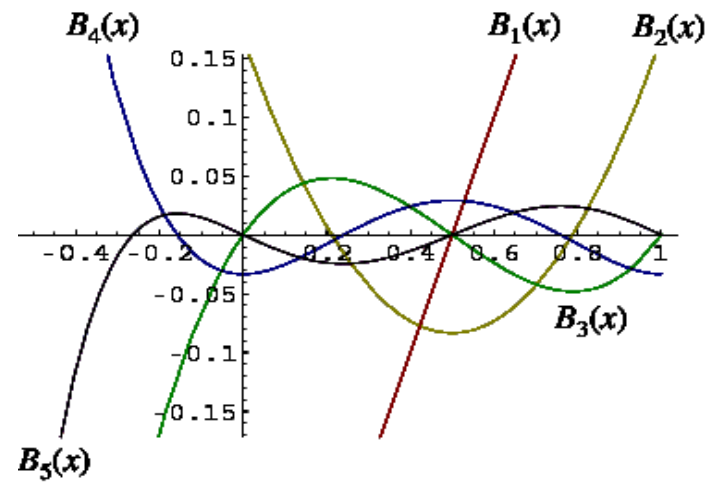

They satisfy $b_{0}(x)=1 \neq 0$, and hence we have a Sheffer set.

For $n=1$,

$$
Q b_{n}=n b_{n-1} \text { becomes } Q b_{1}=1 b_{0}
$$

By Table 1,

$$
Q b_{1}=\alpha_{1} \text { and } 1 b_{0}=1 \Rightarrow \alpha_{1}=1
$$

For $n=2$,

$$
Q b_{n}=n b_{n-1} \text { becomes } Q b_{2}=2 b_{1} \text {. }
$$

From Table 1,

$$
Q b_{2}=2 \alpha_{1} x+\alpha_{2} \text { and } 2 b_{1}=2 x+1 \Rightarrow \alpha_{1}=1 \& \alpha_{2}=1
$$

For $n=3$,

$$
Q b_{n}=n b_{n-1} \text { becomes } Q b_{3}=3 b_{2} \text {. }
$$

By Table 1,

$$
Q b_{3}=3 \alpha_{1} x^{2}+\left(3 \alpha_{2}-3 \alpha_{1}\right) x+\alpha_{3}-\frac{3}{2} \alpha_{2} \quad \text { and } \quad 3 b_{2}=3 x^{2}-\frac{1}{2} .
$$

Equating the corresponding terms, we get

$$
\alpha_{1}=1, \alpha_{2}=1 \& \alpha_{3}=1
$$

For $n=4$,

$$
Q b_{n}=n b_{n-1} \text { becomes } Q b_{4}=4 b_{3}(x) \text {. }
$$

From Table 1, 


$$
Q b_{4}=4 \alpha_{1} x^{3}+\left(6 \alpha_{2}-12 \alpha_{1}\right) x^{2}+\left(4 \alpha_{3}-12 \alpha_{2}+8 \alpha_{1}\right) x+\left(\alpha_{4}-4 \alpha_{3}+4 \alpha_{2}\right)
$$

and

$$
4 b_{3}=4 x^{3}-6 x^{2}+1
$$

Equating the corresponding terms, we get

$$
\alpha_{1}=1, \alpha_{2}=1, \alpha_{3}=1, \& \alpha_{4}=1
$$

Therefore, the characterization of delta operator for the second kind of Bernoulli polynomials being $\alpha_{r}=1$ for all $r \geq 1$.

Thus we obtain the following proposition.

Proposition 2. For the second kind of Bernolli polynomials

$$
b_{n}(x)=\sum_{k=0}^{n}\left(\begin{array}{l}
n \\
k
\end{array}\right) b_{k}(x)_{n-k}
$$

the characterization of delta operator being $\alpha_{r}=1$ for all $r \geq 1$.

(iii) Mott Polynomials. The Mott polynomials $s_{n}(x)$ are defined by

$$
s_{n}(x)=(-1)^{n} \frac{1}{2^{n} x^{n}}(n-1) ! \sum_{k=0}^{h(n / 2)} \frac{x^{-2 k}}{k !(n-k) !(n-2 k-k) !},
$$

where

$$
h(n / 2)=\left\{\begin{array}{ll}
n / 2 & \text { if } n \text { is even } \\
(n / 2)-(1 / 2) & \text { if } n \text { is odd }
\end{array} .\right.
$$

Since $s_{0}(x)=1 \neq 0$, we have a Sheffer set.

The first few Mott polynomials are:

$$
\begin{gathered}
s_{1}(x)=-\frac{1}{2} x \\
s_{2}(x)=\frac{1}{4} x^{2} \\
s_{3}(x)=\frac{-3}{4} x-\frac{1}{8} x^{3} \\
s_{4}(x)=\frac{3}{2} x^{2}+\frac{1}{16} x^{4}
\end{gathered}
$$




$$
\begin{aligned}
& s_{5}(x)=\frac{-15}{2} x-\frac{15}{8} x^{3}-\frac{1}{32} x^{5} \\
& s_{6}(x)=\frac{225}{8} x^{2}+\frac{15}{8} x^{4}+\frac{1}{64} x^{6} .
\end{aligned}
$$

For $n=1, Q s_{n}=n s_{n-1}$ becomes $Q s_{1}=1 s_{0}$.

From Table 1,

$$
Q s_{1}=-\frac{1}{2} \alpha_{1} \text { and } 1 s_{0}=1 \Rightarrow \alpha_{1}=-2 .
$$

For $n=2, Q s_{n}=n s_{n-1}$ becomes $Q s_{2}=2 s_{1}$.

By Table 1,

$$
Q s_{2}=\left(\frac{1}{2} \alpha_{1}\right) x+\frac{1}{2} \alpha_{2} \text { and } 2 s_{1}=(-1) x \Rightarrow \alpha_{1}=-2 \& \alpha_{2}=0 .
$$

For $n=3, Q s_{n}=n s_{n-1}$ becomes $Q s_{3}=3 s_{2}$. From Table 1,

$$
Q s_{3}=\left(-\frac{3}{8} \alpha_{1}\right) x^{2}+\left(-\frac{3}{8} \alpha_{2}\right) x+\left(-\frac{3}{4} \alpha_{1}-\frac{1}{8} \alpha_{3}\right) \text { and } 3 s_{2}=\left(\frac{3}{4}\right) x^{2} .
$$

Equating the corresponding terms, we get

$$
\alpha_{1}=-2, \alpha_{2}=0 \& \alpha_{3}=12 .
$$

For $n=4, Q s_{n}=n s_{n-1}$ becomes $Q s_{4}=4 s_{3}$. From Table 1,

$$
Q s_{4}=\left(\frac{1}{4} \alpha_{1}\right) x^{3}+\left(\frac{8}{3} \alpha_{2}\right) x^{2}+\left(3 \alpha_{1}+\frac{1}{4} \alpha_{3}\right) x+\left(\frac{3}{2} \alpha_{2}+\frac{1}{16} \alpha_{4}\right)
$$

and

$$
4 s_{3}=(-3) x+\left(-\frac{1}{2}\right) x^{3} .
$$

Comparing the corresponding terms, we get

$$
\alpha_{1}=-2, \alpha_{2}=0 \alpha_{3}=12 \& \alpha_{4}=0 .
$$

For $n=5, Q s_{n}=n s_{n-1}$ becomes $Q s_{5}=5 s_{4}$. By Table 1, finding values for $Q s_{5} \& 5 s_{4}$ and equating the corresponding terms, we get

$$
\alpha_{1}=-2, \alpha_{2}=0 \alpha_{3}=12 \alpha_{4}=0 \& \alpha_{5}=-240 .
$$

Applying the same procedure for $n=6, n=7$ and $n=8$, we get

$$
\begin{array}{ll}
\alpha_{1}=-2=-2(1 !) & \alpha_{2}=0 \\
\alpha_{3}=12=2(3 !) & \alpha_{4}=0 \\
\alpha_{5}=-240=-2(5 !) & \alpha_{6}=0 \\
\alpha_{7}=10080=2(7 !) & \alpha_{8}=0 .
\end{array}
$$


Thus we have the following proposition.

Proposition 3. For the Mott polynomials

$$
s_{n}(x)=(-1)^{n} \frac{1}{2^{n} x^{n}}(n-1) ! \sum_{k=0}^{h(n / 2)} \frac{x^{-2 k}}{k !(n-k) !(n-2 k-k) !},
$$

the characterization of delta operator being

$$
\alpha_{r}= \begin{cases}0 & \text { if } r \text { is even } \\ (-1) 2(r !) & \text { if } r=1,5,9, \cdots \\ 2(r !) & \text { if } r=3,7,11, \cdots\end{cases}
$$

From the above discussion, we get a way opened to study the Sheffer polynomials by a new approach of finding definite delta operator numerically. All the results are shown vividly in the following table.

\begin{tabular}{|c|l|}
\hline Polynomials & Characterization of Delta Operator \\
\hline Euler & $\alpha_{1}=1$ and $\alpha_{r}=0$, for all $\mathrm{r} \geq 2$. \\
\hline Bernoulli II & $\alpha_{r}=1 \quad r \geq 1$. \\
\hline Mote & $\alpha_{r}=0$ if $r$ is even \\
& $\alpha_{r}=(-1) 2(r !)$ if $r=1,5,9 \cdots$ \\
& $\alpha_{r}=2(r !)$ if $r=3,7,11 \cdots$ \\
\hline
\end{tabular}

Table 2. Delta operators for Different Sheffer polynomials

\section{Status and Further Directions}

An attempt is made to introduce a new approach to the Sheffer polynomials via sequential representation of the delta operator. The basic polynomial sequence in $q$-monodiffric sense with its $q$-delta operator is discussed and analyzed in Maheswaran [7]. This is the good starting point for further investigation of the characterization of the $q$-delta operator for $q$-Sheffer polynomials.

\section{References}

[1] R.P. Boas and R.C. Buck, Polynomial Expansion of Analytic Functions, Springer-Verlag, New York (1964). 
[2] Dae San Kim, Taekyun Kim, Seog-Hoon Rim and D.V. Dolgy, Sheffer sequences of polynomials and their applications, Advances in Difference Equations, 2013 (2013), Article \# 118, 10 p.

[3] G. Dattoli, M. Migliorati and H.M. Srivastava, Sheffer polynomials, monomiality principle, algebraic methods and the theory of classical polynomials, Mathematical and Computer Modelling 45 (2007), 1033-1041.

[4] Do Tan Si, Differential calculus for differential equations, special functions, Laplace transform, Canadian Center of Science and Education, Applied Physics, 8, No 1 (2016), 158-187.

[5] Feng Qi, Dae San Kim, Tae-Kyun Kim and V. Dolgy, Multiple-polyBernoulli polynomials of the second kind, Advanced Studies in Contemporary Mathematics, 25, No 1 (2015), 1-7.

[6] A. Maheswaran and C. Elango, Sequential representation of delta operator in finite operator calculus, British Journal of Mathematics and Computer Science, 14, No 2 (2016), 1-11.

[7] A. Maheswaran, Extension of Finite Operator Calculus to q-Monodiffric theory, M.Ph. Dissertation, Bharathiyar University, Coimbatore, Tamil Nadu - India (1988).

[8] E.Di. Nardo, H. Niederhausen and D. Senato, A symbolic handling of Sheffer polynomials, Annali di Mathematica, 190 (2011), 489-506.

[9] T.R. Prabhakar and S. Gupta, Bernoulli polynomials of the second kind and general order, Indian J. Pure Appl. Math., 11, No 10 (1980), 13611368.

[10] E.D. Rainville, Special Functions, Chalsea Publishing Company (1960).

[11] G.C. Rota, Finite Operator Calculus, Academic Press, London (1975).

[12] Salahuddin and I. Husain, Computation of some wonderful results involving certain polynomials, Intern. Res. J. of eng. and Technology 02, No 01 (2015), 382-391.

[13] Taekyun Kim, Hyuck In Kwon, Sang Hun Lee and Jong Jin Seo, A note on poly-Bernoulli numbers and polynomials of the second kind, Advances in Difference Equations 2014, Article \# 219, 6 p. 
[14] Veli Kurt, New identities and relations derived from the generalized Bernoulli polynomials, Euler polynomials and Genocchi polynomials, $A d$ vances in Difference Equations 2014 (2014), Article \# 5, 9 p.

[15] Wolfram Mathworld (October 2013), Mott Polynomial, Retrived from website:

http://mathworld.wolfram.com/MottPolynomial.html.

[16] Zhi-Wei Sun and Hao Pan, Identities concerning Bernouli and Euler Polynomials, Acta Arith. 125 (2006), No 1, 21-39. 